\title{
An Automatic Selection Method for the Visual Scene LOD Model and Viewpoint based on Genetic Algorithm and Image Entropy
}

\author{
Xiaodong Wang ${ }^{1,2, a^{*}}$, Hao $\mathrm{Gu}^{1,2, \mathrm{~b}}$ and Fengju Kang ${ }^{1,2, \mathrm{c}}$ \\ ${ }^{1}$ School of Marine Science and Technology, Northwestern Polytechnical University, Xi'an, China \\ ${ }^{2}$ National Key Laboratory of Underwater Information Process and Control, Xi'an, China \\ awangxiaodong@mail.nwpu.edu.cn, ’bwuniorbrian@126.com, kangfengju@nwpu.edu.cn
}

\section{Keywords: Genetic algorithm, Automatic selection, LOD model, Viewpoint, Image entropy}

Abstract. The development of 3D real-time visual scenes is usually a time-consuming manual process due to its massive models and viewpoint layout. As a result, an automatic selection method for the visual scene level of detail (LOD) model and viewpoint based on genetic algorithm (GA) and image entropy is proposed in this paper. Image entropy is an objective index for evaluation, which makes automatic selection possible. The method comprises three steps: obtaining the model set and the viewpoint set according to the analysis of scene elements; searching for an optimal combination scheme of the scene through GA; rendering the optimal scene. The experiment result shows that the method proposed above effectively determines the model level and viewpoint, which can be used to the visual scene development.

\section{Introduction}

With the continuous development of computer technology, the three-dimensional visual scene simulation technology is widely used in scientific research, military training, teaching, entertainment and many other fields. It is a comprehensive application of computer graphics, image processing technology, multimedia technology, three-dimensional display technology and other high technologies. Its main task is to establish the simulation model with high fidelity and complete the real-time rendering of $3 \mathrm{D}$ scene.

The fidelity and real-time performance are important indexes of visual scene. Generally, a more detailed three-dimensional model can represent the simulation object more realistically. However, establishing the detailed three-dimensional model needs more triangles, which would increase burden on the computer and reduce the scene quality. Therefore the real-time performance would be reduced. In order to solve the contradiction between real-time performance and fidelity, Luebke proposed the concept of LOD which needs the developers to determine the level of detail based on the model's position and importance in the scene [1]. In the process of developing real-time scene, the developers usually need to choose an appropriate viewpoint by several attempts to make the scene information reach to the maximum.

Zeng researched the combinatorial optimization of 3D visualization system based on automatic-correction, which applied Particle Swarm Optimization (PSO) to obtain optimal rendering effect [2]. Gotovac presented a novel multi-resolution adaptive Fup approach, the approach is and efficient particularly when describing sharp fronts and narrow transition zones changing in space and time [3]. Zhang presented a multi-scale and multi-resolution agent-based in silico glioma model, to reduce the computation time of a discrete-based model substantially [4]. Yang presented two kinds of resolution model on pedestrian evacuation and simulation. One is based on Multi-Agent framework and a modified PSO algorithm. The other is based on the Euler equations of fluid dynamics [5]. These methods produce the visual mappings which are from the real world to models, and it can be easily understood by the model developer. But, there are also many problems such as temporal inconsistency, chain disaggregation and so on. 
Considering the two artificial selection processes proposed above is time-consuming and cannot achieve the best results, we sample images when the viewpoint is at each valid position and the model is at all the levels, and choose the image entropy as fitness value of GA to find the optimal viewpoint and LOD model level.

\section{The Process of Automatic Selection}

Before discussing the method, we introduce two important theories. The first one is image entropy which was first introduced by John Zachary to describe the 'business' of an image, i.e. the amount of information which must be coded for by a compression algorithm [6]. The image entropy is the defined in Eq. 1.

$$
E(I M G)=-\sum_{i=1}^{n} h_{i} \log _{2}\left(h_{i}\right)
$$

where $h_{i}$ is the probability that the color value of the pixel is equal to $i$. If we take the grayscale image as probability density function, the image entropy can be expressed as Eq. 2.

$$
\begin{aligned}
& E(I M G)=-\sum_{i=1}^{\text {width height }} \sum_{j=1}^{\text {width height }} p(i, j) \log _{2} p(i, j) \\
& p(i, j)=x(i, j) / \sum_{i=1}^{\text {he }} \sum_{j=1} x(i, j)
\end{aligned}
$$

where width and height denote the width and height of the image respectively, $x(i, j)$ is the image grayscale at the position $x(i, j)$. Therefore, we need to convert color image to grayscale image when calculating its entropy value. For the color image in RGB format, the grayscale is given according to the ratio RED: GREEN: BLUE = 0.3: 0.59: 0.11 as shown in Eq. 3.

$$
x(i, j)=0.3 * R(i, j)+0.59 * G(i, j)+0.11 * B(i, j)
$$

Another theory related to this paper is GA that has become a mature optimization algorithm with adaptive parallelism, wide applicability and strong robustness. Therefore, we apply GA to the automatic selection of visual scene LOD model and viewpoint. The solution process is shown as Fig. 1. 


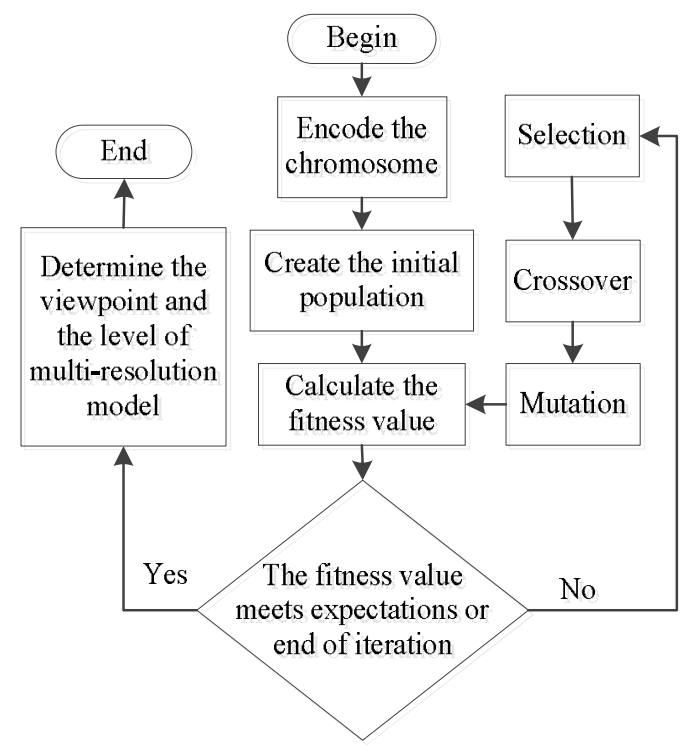

Fig. 1. The structure of solution process flow.

Encoding the Model Set and Viewpoint Set. We assume that there are n models in the visual scene and their levels are respectively $L_{1}, L_{2}, \ldots, L_{n}$, and then get kinds of combination scheme. Due to the limitations of the computer, it usually disobeys the fact that we can obtain the best scheme if all the models with highest resolution are selected. That is to say the image entropy related to this scheme is not the highest.

Definition 1. The model set consists of all the LOD model combination schemes, denoted by $\Psi$.

$$
\Psi=\left\{m_{1}, m_{2}, m_{3}, \ldots, m_{n} \mid m_{1} \in\left\{1,2, \ldots, L_{1}\right\}, m_{2} \in\left\{1,2, \ldots, L_{2}\right\} \ldots, m_{n} \in\left\{1,2, \ldots, L_{n}\right\}\right\}
$$

In the usual development work, the viewpoint selection is realized by artificial observation, which relates to the evaluation problem of viewpoint equality. As shown in Fig. 2, we observe the same scene in different viewpoints, Fig. 2(a) shows more details compared to Fig. 2(b), and therefore the former viewpoint is considered more reasonable.

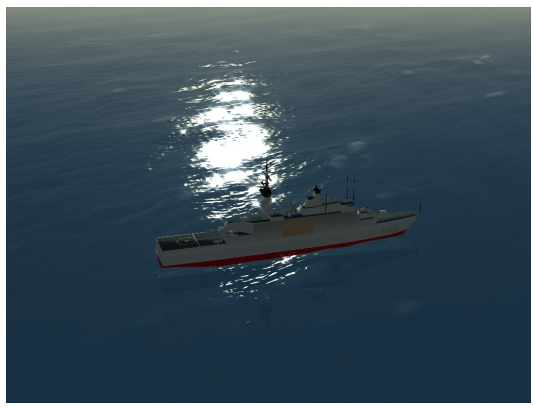

(a)

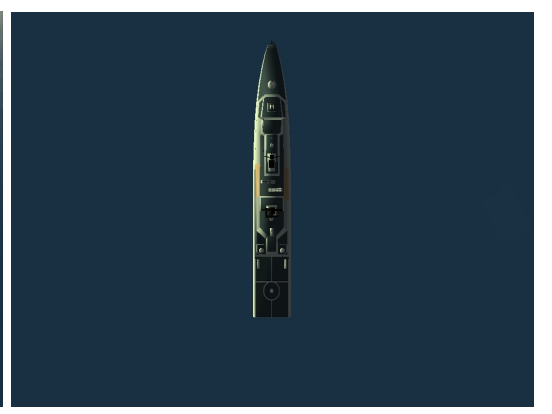

(b)

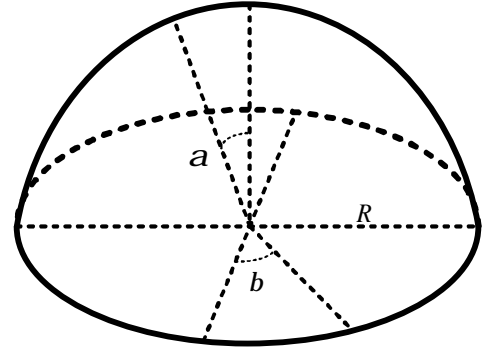

Fig. 3. The hemispherical surface.

Fig. 2. The rendering image in different viewpoints.

The best viewpoint selection relates to the density of the considered viewpoints (including the distance and azimuth parameters), which is implemented by evaluating every viewpoint with a certain method. The efficiency of this process is decided by the sampling density of viewpoint. We sample on the hemispherical surface shown in Fig. 3, define the sampling points as viewpoint set denoted by , divide the surface into $\mathrm{P}$ equal segments with interval along the Longitude direction, and $\mathrm{Q}$ equal segments with interval along the latitude direction. Then the model set size is $P \times Q$.

$$
\begin{aligned}
& \Omega=\left\{(R \sin \alpha \sin \beta, R \sin \alpha \cos \beta, R \cos \alpha) \mid \alpha \in\left\{\alpha_{1}, \alpha_{2} \cdots \alpha_{Q}\right\}, \beta \in\left\{\beta_{1}, \beta_{2} \cdots \beta_{P}\right\}\right\} \\
& \alpha_{1}=0, \alpha_{2}=\operatorname{step} 2, \cdots, \alpha_{i}=i \cdot \text { step } 2, \cdots, \alpha_{Q}=0.5 \pi \beta_{1}=0, \beta_{2}=\text { step } 1, \cdots, \beta_{i}=i \cdot \text { step } 1, \cdots, \beta_{P}=2 \pi
\end{aligned}
$$


Definition 2. Assume that $\psi(\omega)$ is an element from the model set (viewpoint set), and $(\psi, \omega)$ is one combination scheme of the model and viewpoint, define all the schemes as model and viewpoint set denoted by $\Gamma$, its size $I=L_{1} \times L_{2} \times \ldots \times L_{n} \times P \times Q$.

$$
\Gamma=\{(\psi, \omega) \mid \psi \in \Psi, \omega \in \Omega\}
$$

The set $\Gamma$ is our optimization space with GA, and we use a binary string of length $S$ to represent one combination scheme which is also called the individual in GA. It is necessary that $S$ is no lesser than $\log _{2} I$.

The Fitness Calculation. GA needs a fitness function to describe the species' adaption capability to the environment. Since the fitness of the individual is measured by the entropy value of images which are sampled from the visual scene in all schemes. The fitness calculation is converted into querying the corresponding entropy value. Therefore, it is necessary to build a query table as shown in Table 1 for all the image entropy values.

Table 1. LOD model combination scheme and entropy value.

\begin{tabular}{ccc}
\hline Index & Combination Scheme $\beta \times \alpha \times L_{n} \times \cdots \times L_{1}$ & Entropy value \\
\hline 0 & $00 \ldots 00$ & $f_{0}$ \\
1 & $00 \ldots 01$ & $f_{1}$ \\
$\cdots$ & $\ldots$ & $\cdots$ \\
$I$ & $(P-1)(Q-1)\left(L_{n}-1\right)\left(L_{n-1}-1\right) \cdots\left(L_{1}-1\right)$ & $f_{I}$ \\
\hline
\end{tabular}

Selection, Crossover and Mutation. Selection is the process that the fittest survive to pass their genes on to the next generation and the least are eliminated. The individuals with higher fitness value have more probability to be chosen, and the number of their descendants is greater. Then the individuals selected are put into the mating pool. We choose the roulette wheel model in this paper. Assuming that the population size is $n$, individual fitness value is $f_{i}$, and then the possibility that individual $i$ is selected $P_{s i}=f_{i} / \sum_{i=1}^{n} f_{i}$.

The process that two individuals, which are randomly selected from the mating pool, exchange a segment of gene string according to the crossover rate $P_{c}$ is called crossover, which plays the key role in producing new individuals. Mutation operator randomly changes the chromosome gene values with a tiny mutation rate $P_{m}$.

\section{Experimental Results}

Our aim in this section of the paper is to apply the automatic selection method proposed and analysis the effectiveness. First we develop a simple visual scene that shows a warship swimming in the sea, then calculate the entropy values of all the images which are sampled from the visual scene in all the schemes, and finally use the GA to obtain the optimal scheme. The warship LOD model has five levels resolution, and the corresponding number of faces and triangles of each level is shown in Table 2. The experiment is designed with the GA parameter setting: population size $n=30$, gene string length $S=9$, crossover rate $P_{c}=0.65$, mutation rate $P_{m}=0.1$, and the viewpoint hemispherical surface parameter setting: radius $R=245 \mathrm{~m}, P=Q=10, I=P \times Q \times L_{1}=5 \times 10 \times 10=500$. 
Table 2. Warship LOD model geometric parameters.

\begin{tabular}{ccc}
\hline Level of Detail & Triangles & Faces \\
\hline 1 & 1016 & 565 \\
2 & 1824 & 953 \\
3 & 2286 & 1237 \\
4 & 3934 & 2043 \\
5 & 4610 & 2385 \\
\hline
\end{tabular}

With the process of evolution, the population shows more and more strong adaptability. Fig. 4 shows all individuals' fitness from the first, fourth, sixth and tenth generation. The curve denotes the change trend of fitness corresponding to the 500 combination schemes, and the asterisks in each sub-image denote fitness of the 30 individuals from that generation. The phenomenon that population fitness converges to the maximum indicates that population adaptability develops towards the stronger direction.
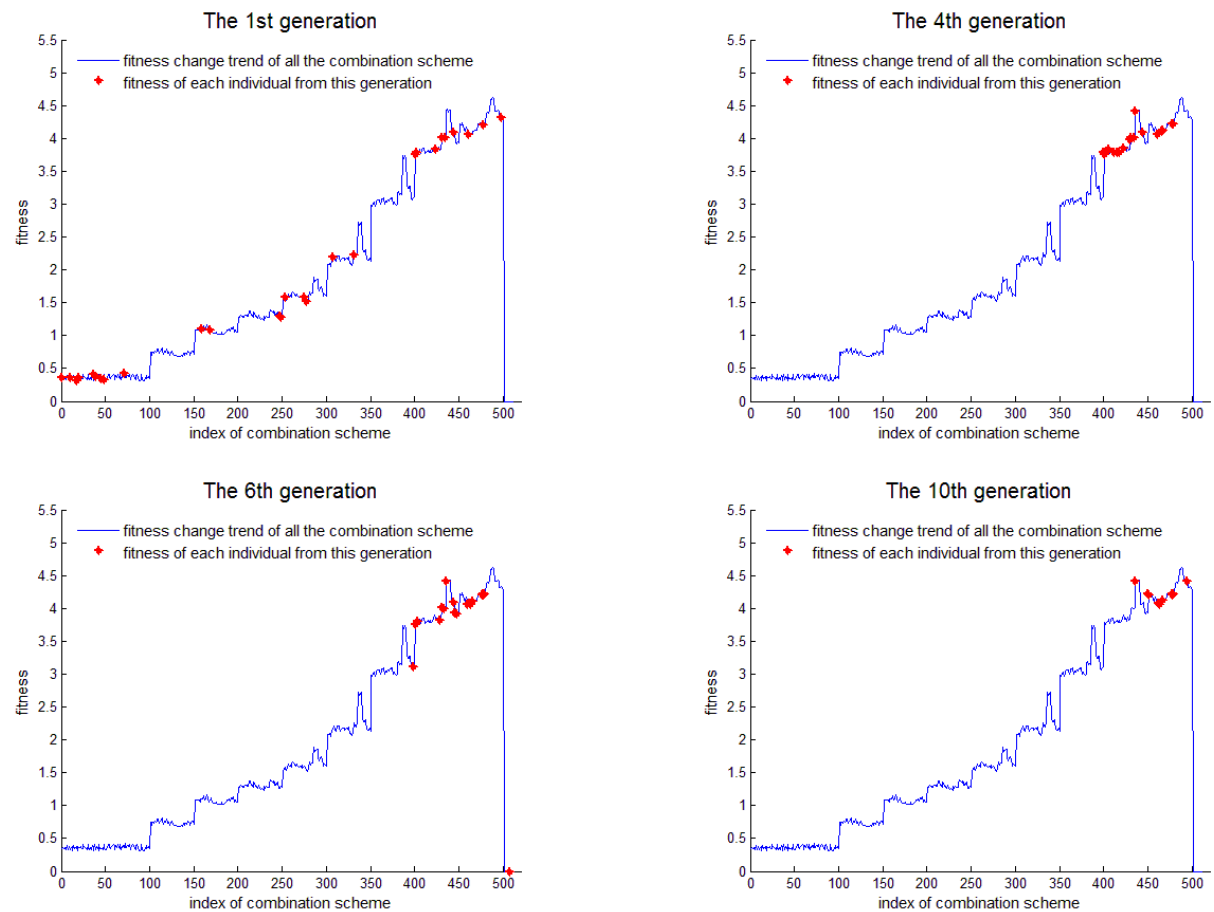

Fig. 4. Change trend of individuals from four generations.

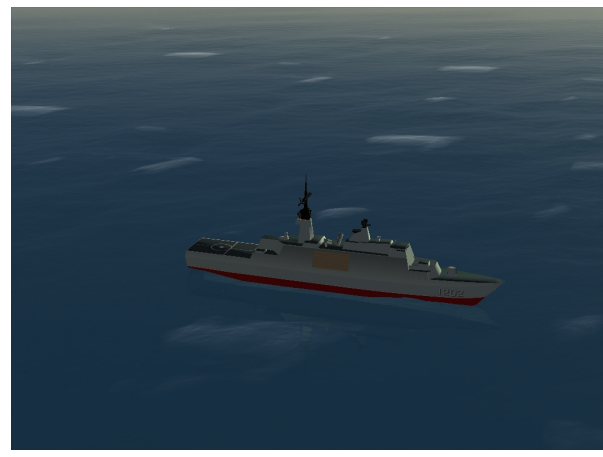

(a) The 1 st generation $(8 \times 6 \times 4)$

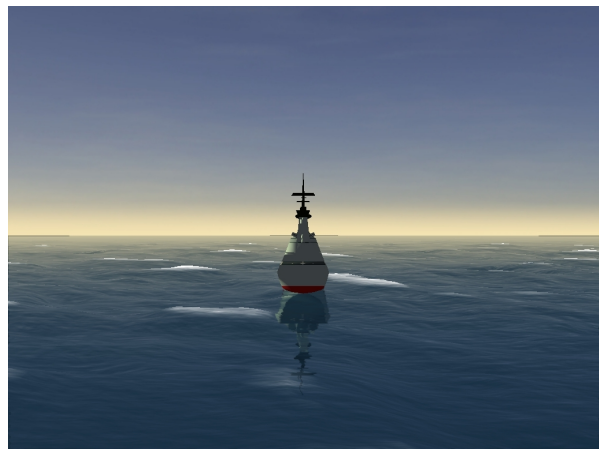

(b) The 4th generation $(9 \times 0 \times 3)$ 


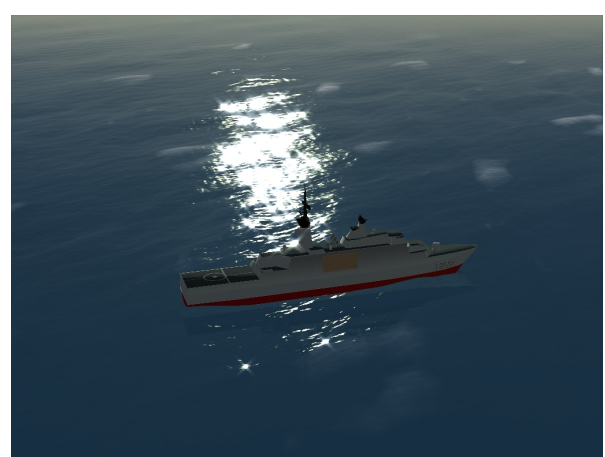

(c) The 6th generation $(9 \times 6 \times 3)$

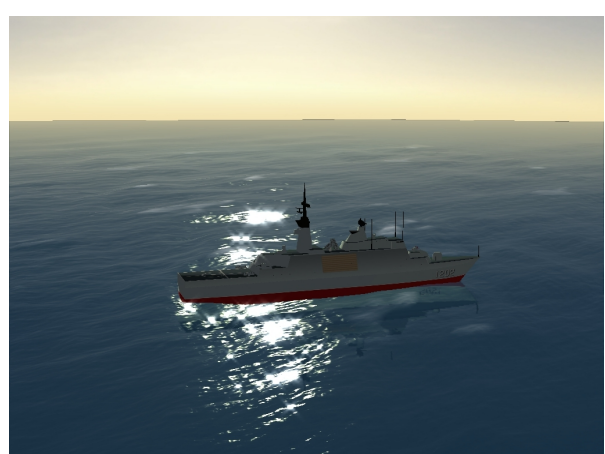

(d) The 10th generation $(9 \times 7 \times 1)$

Fig. 5. The optimal visual scenes corresponding to the best individual.

The optimal visual scenes corresponding to the best individuals from the four generations above are shown in Fig. 5. Considering the visual scene information such as: sky, light effect and warship reflection in the sea surface, we can make the conclusion that Fig. 5(d) has the best visual effect. Therefore, the optimal combination scheme is $9 \times 7 \times 1$, which corresponds to the first level of warship LOD model and the viewpoint $(9 \times \pi / 5,7 \times \pi / 20)$.

\section{Conclusions}

This work has proposed an automatic selection method for the visual scene LOD model and viewpoint based on GA and image entropy, which solves the following problems: (1) the time-consuming artificial selection process; (2) the relatively poor optimization effect. The experiment result shows that this method can effectively select the optimal one from huge number of combination schemes. What needs to be pointed out is that the optimal scheme we mentioned is relatively more close to the theoretical optimal results when viewpoint set sampling density and discrete model resolution are considered.

\section{References}

[1] D. Luebke and C. Erikson, View-dependent simplification of arbitrary polygonal environments, SIGGRAPH 97 Proceedings of the 24th annual conference on Computer graphics and interactive techniques, pp.199-208, 1997.

[2] Y Y Zeng. Combinatorial optimization of three-dimensional visualization simulation based on automatic-correction and its application in virtual battlefield environment, Xi' an, China: Northwestern Polytechnical University, 2014.

[3] H Gotovac, R Andricevic, B Gotovac. Multi-resolution adaptive modeling of groundwater flow and transport problems, Advances in Water Resources(0309-1708), 2007, 30(5): 1105-1126.

[4] L Zhang, L Leon, T S Deisboeck. Multi-scale, multi-resolution brain cancer modeling [J]. Mathematics and Computers in Simulation(0378-4754), 2009, 79(7): 2021-2035.

[5] B Yang, B Ren, Y G Wu. The Research of Multi-Resolution Modeling and Simulation of the Emergency Evacuation, 2012 International Workshop on Information and Electronics Engineering, 2012, 29: 3110-3116.

[6] J. M. Zachary, An Information Theoretic Approach to Content Based Image Retrieval, Phd, Thesis Louisiana State University and Agricultural \&Mechanical College, pp.45-62, 2000. 